\title{
Benefits of microscopy with super resolution
}

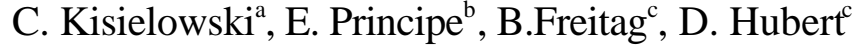

${ }^{a}$ National Center for Electron Microscopy, Ernest Orlando Lawrence Berkeley National Laboratory, Berkeley, CA 94720, USA, Fax: (510) 486 5888, E-mail: CFKisielowski@lbl.gov

${ }^{\mathrm{b}}$ Applied Materials, 3050 Bowers Avenue, Santa Clara, CA 95054, USA

${ }^{\mathrm{c}}$ FEI Company, Achtseweg Noord 5, P.O. Box 218, 5600 MD Eindhoven, The Netherlands

\begin{abstract}
Transmission Electron Microscopy developed from an imaging tool into a quantitative electron beam characterization tool that locally accesses structure, chemistry, and bonding in materials with sub Angstrom resolution. Experiments utilize coherently and incoherently scattered electrons. In this contribution, the interface between gallium nitride and sapphire as well as thin silicon gate oxides are studied to understand underlying physical processes and the strength' of the different microscopy techniques. An investigation of the GaN/sapphire interface benefits largely from the application of phase contrast microscopy that makes it possible to visualize dislocation core structures and single columns of oxygen and nitrogen at a closest spacing of 85 pm. In contrast, it is adequate to investigate $\mathrm{Si} / \mathrm{SiO}_{\mathrm{x}} \mathrm{N}_{\mathrm{y}} /$ poly-Si interfaces with incoherently scattered electrons and electron spectroscopy because amorphous and poly crystalline materials are involved. Here, it is demonstrated that the $\mathrm{SiO}_{\mathrm{x}} \mathrm{N}_{\mathrm{y}} /$ poly-Si interface is rougher than the $\mathrm{Si} / \mathrm{SiO}_{\mathrm{x}}$ interface, that desirable nitrogen diffusion gradients can be introduced into the gate oxide, and that a nitridation coupled with annealing increases its physical width while reducing the equivalent electrical oxide thickness to values approaching $1.2 \mathrm{~nm}$. Therefore, an amorphous $\mathrm{SiN}_{\mathrm{x}} \mathrm{O}_{\mathrm{y}}$ gate dielectric seems to be a suitable substitute for traditional gate oxides to further increase device speed by reducing dimensions in Si technology.
\end{abstract}

Keywords: Transmission Electron Microscopy, Gallium nitride, Gate oxides, Dislocations 


\section{DISCLAIMER}

This document was prepared as an account of work sponsored by the United States

Government. While this document is believed to contain correct information, neither the United States Government nor any agency thereof, nor The Regents of the University of California, nor any of their employees, makes any warranty, express or implied, or assumes any legal responsibility for the accuracy, completeness, or usefulness of any information, apparatus, product, or process disclosed, or represents that its use would not infringe privately owned rights. Reference herein to any specific commercial product, process, or service by its trade name, trademark, manufacturer, or otherwise, does not necessarily constitute or imply its endorsement, recommendation, or favoring by the United States Government or any agency thereof, or The Regents of the University of California. The views and opinions of authors expressed herein do not necessarily state or reflect those of the United States Government or any agency thereof, or The Regents of the University of California. Ernest Orlando Lawrence Berkeley National Laboratory is an equal opportunity employer. 


\section{Background}

Recent progress in High Resolution Transmission Electron Microscopy (HRTEM) allows investigating crystalline materials by phase contrast microscopy with a resolution close to the 80 pm information limit of a $300 \mathrm{kV}$ field emission microscope ${ }^{1-4)}$. A reconstruction of the electron exit wave from a focal series of lattice images converts the recorded information into interpretable resolution ${ }^{5,6)}$. It was reported before that Z-contrast microscopy achieved sub Angstrom resolution ${ }^{7)}$.

From a materials point of view, a resolution enhancement in electron microscopy is required to achieve truly mono atomic resolution in most materials systems. A typical point resolution around $0.2 \mathrm{~nm}$ does commonly not suffice. Figure 1 depicts this situation by plotting the band gap energy of different ceramics and semiconductors versus their inter atomic spacing. It is seen that the resolution of single atomic columns in ceramic-like materials is barely possible. In practice, their separation often requires a better point resolution because lattice imaging along zone axes of crystals nearly always shortens the inter atomic spacing by the involved projection into the image plane. For example, the resolution of dumbbell images along the commonly recorded [110] zone axis of silicon, cubic gallium nitride, and diamond require a point resolution of $0.136 \mathrm{~nm}, 0.113 \mathrm{~nm}$, and $0.089 \mathrm{~nm}$, respectively.

Moreover, band gap energies tend to increase with shorter inter atomic distances. From the slope of the trend line in figure 1 one finds that a reduction by 1 pm typically changes energies by 50 $\mathrm{meV}$. It is for such reasons that electron microscopy strives for the development of equipment that will allow for sub Angstrom resolution ${ }^{3,4,7)}$, will pinpoint the position of atomic columns to within $1 \mathrm{pm}^{12,13)}$, and will enable spectroscopic investigations of single atomic columns with an energy resolution of about $50 \mathrm{meV}[8,9,10,11]$.

In the present contribution we investigate interfaces with current state-of-the-art equipment. A CM300 FEG/UT HRTEM with an information limit of $80 \mathrm{pm}$ and a Tecnai F20/ST HRTEM/STEM (Scanning Transmission Electron Microscope) with a beam diameter of 0.19 $\mathrm{nm}, 500 \mathrm{meV}$ of energy resolution, and Z-contrast capability are employed. This allows for a comparison of phase - with Z-contrast imaging. Gate oxides in silicon and the interface between 
GaN and sapphire are examined. Both examples are of substantial technological importance ${ }^{14,15)}$. Details of sample preparation procedures are published elsewhere ${ }^{3)}$.

\section{Gate oxides}

Figure 2 depicts images of the amorphous $\mathrm{SiO}_{2}$ gate dielectric of roughly $2 \mathrm{~nm}$ thickness that was deposited on silicon and capped with a poly-silicon layer. Gate oxides of this thickness will be relevant for a $0.1 \mu \mathrm{m}$ technology that must be standard technology in a few years if Moore's law applies. Excellent film uniformity has to be achieved across wafers and TEM is the primary tool to relate the physical gate oxide thickness to process parameters and to calibrate metrology tools.

We depict three types of images in figure 2: Figure 2a is a usual lattice image which is an interferogram of diffracted beams ${ }^{16)}$. As a result the image pattern of the silicon and the polysilicon changes rapidly with the focus setting of the objective lens that also acts as a filter for spatial frequencies. This produces the speckle contrast of the amorphous material. In addition, there is substantial delocalization of information at interfaces that is visible as a periodic contrast variation in the amorphous layer close to the silicon substrate. It results from the utilization of a field emission electron source ${ }^{16)}$. Figure $2 b$ is a reconstructed phase image of the electron exit wave from a focal series of lattice images ${ }^{3,5,6)}$. Delocalization effects are removed during the reconstruction process. Poly-crystalline grains are visible even if they are not imaged along low indexed zone axes because of the 80 pm information limit of the instrument. In thin samples $(<$ $10 \mathrm{~nm}$ ) the image depicts the $1 \mathrm{~s}$ state of the electron wave channeling along atomic columns ${ }^{17)}$ that are entirely resolved. They appear as bright spots in the phase image. The defocus dependence of the pattern is eliminated. Local phase changes can be analyzed quantitatively since they oscillate with sample thickness. Their oscillation periodicity is given by ${ }^{17)}$ :

$\mathrm{D}_{1 \mathrm{~s}}=\alpha *\left[\mathrm{~d}^{2} / \mathrm{Z}+0.276 * \mathrm{~B}\right]$

where $\alpha$ is a constant, $\mathrm{d}$ is the distance between successive atoms, $\mathrm{Z}$ is the atomic number and $\mathrm{B}$ is the Debye Waller factor. Equation (1) was recently utilized to demonstrate that the phase change at single gold atoms can be detected with a signal to noise ratio of better than $2^{18)}$. Thus, unlike a lattice image, the phase image of the electron exit wave depicts directly the projection of 
through a simple dependence on sample thickness and chemical composition. However, the speckle contrast of the amorphous $\mathrm{SiO}_{2}$ remains because the reconstruction process operates over the finite range of spatial frequencies that is determined by the objective lens ${ }^{5,6}$. Finally, Figure $2 \mathrm{c}$ depicts a Z-contrast image. Similarly to the phase images, a Z-contrast image also depicts the 1s state of electrons trapped on atomic columns. However, incoherently scattered electrons are used to build up the picture ${ }^{19)}$. Currently, sub Angstrom resolution is achievable by Z-contrast microscopy at $300 \mathrm{kV}^{7)}$. However, the $0.136 \mathrm{~nm}$ separation of the dumbbell structure in silicon can already be resolved at $200 \mathrm{kV}^{20)}$. In comparison with phase contrast images, Zcontrast images lack of speckle contrast in amorphous regions. Its absence eases a quantitative evaluation of intensity profiles to measure the gate oxide thickness and interfacial roughness ${ }^{21)}$.

Table 1 summarizes results of gate oxide thickness measurements from Z-contrast images and from the phase contrast images. Intensities were evaluated in Z-contrast images and the presence of a pattern from crystalline material was used to locate the position of interfaces in phase contrast images. Two samples were investigated: a control sample (A) and a second sample (B) where nitrogen was diffused into the gate oxide layer. From a comparison of gate oxide thickness' in sample A and B one finds that a broadening of the amorphous region occurs as a result of nitrogen incorporation. Moreover, we find that the poly-Si/SiO 2 interface is rougher by a factor of 2 than the $\mathrm{Si} / \mathrm{SiO}_{2}$ one. Finally, phase contrast images consistently provide a smaller gate oxide thickness than measurements obtained from $\mathrm{Z}$-contrast images.

We recorded Electron Energy Loss Spectra (EELS) by stepping the focused $(0.19 \mathrm{~nm})$ electron across the interfaces with $0.1 \mathrm{~nm}$ per step. At each step, a full spectrum including the $\mathrm{Si} \mathrm{L}_{23}, \mathrm{~N}$ $\mathrm{K}$, and $\mathrm{O} \mathrm{K}$ edges was recorded. Post experiment, we processed the several thousand spectra to produce chemical maps or profiles as shown in figure 3. Oxygen, nitrogen and silicon profiles are depicted and the thickness values of the gate oxide as determined from the Z-contrast - and phase contrast images are overlaid. The profiles reveal that $\mathrm{O}$ and $\mathrm{N}$ gradients are present in the gate oxide. Nitrogen concentrations peak at the poly- $\mathrm{Si} / \mathrm{SiO}_{\mathrm{x}} \mathrm{N}_{\mathrm{y}}$ interface and decrease linearly towards the $\mathrm{Si} / \mathrm{SiO}_{\mathrm{x}}$ interface. It is not surprising to find that the width determination of the gate dielectric from the Z-contrast images coincides with its chemical width as measured by EELS since the intensities in a Z-contrast image depend on sample thickness and on chemistry. 
In summary, we produced a direct comparison between lattice images, electron exit waves, and Zcontrast images of gate oxides. In silicon [110] a resolution of $136 \mathrm{pm}$ suffices to separate individual atomic columns at $300 \mathrm{kV}$ by coherently scattered electrons in combination with exit wave reconstruction and by incoherently scattered electrons. Even the current information limit of leading edge $200 \mathrm{kV}$ equipment allows for a resolution of this dumbbell structure. Both techniques provide pictures of the 1s state of electrons trapped at atomic columns and the signal strength depends on local sample thickness and chemical composition. Differences stem from a larger signal to noise ratio in phase contrast images compared with Z-contrast images. However, in the presence of amorphous material, the limited bandwidth of transmitted spatial frequencies in phase contrast microscopy generates a speckle contrast in amorphous - and partly in polycrystalline material. Its absence in Z-contrast images eases a quantitative evaluation of interfacial roughness and the dielectric layer width. A nitridation of the gate oxide increases its dielectric constant and the layer width. Both effects are beneficial for devices because they appear electrically "thinner" but are physically thicker. The equivalent electrical thickness approaches $1.2 \mathrm{~nm}$ in this case. Therefore, it is feasible to further increase the switching speed of devices while avoiding tunnel currents. A control of nitrogen diffusion gradients is crucial in this process and electron spectroscopy can provide this information on a scale of $0.1-0.2 \mathrm{~nm}$. From a comparison of phase contrast images, $\mathrm{Z}$-contrast images, and electron spectroscopy we find that $\mathrm{O}$ and $\mathrm{N}$ diffused into the adjacent crystalline materials.

\section{Growth of GaN on sapphire}

It is evident from figure 1 that an investigation of silicon is not extremely demanding in terms of resolution. In case of $\mathrm{GaN}$ or sapphire $\left(\mathrm{Al}_{2} \mathrm{O}_{3}\right)$, however, the situation changes for two reasons: the inter atomic distances are smaller and the light elements $\mathrm{O}$ and $\mathrm{N}$ are involved with low electron scattering power. It is for these reasons that these materials could not be investigated on a truly atomic scale.

Commonly, lattice images of $\mathrm{GaN}$ are recorded along a [11ㅡㄹ zone axis as shown in figure 4a because it is this projection that allows at least for a separation of the Ga atoms. Even more restricting is the presence of a huge defect density at the interface between GaN and sapphire 
region in [1100] projection. This experiment benefits from two factors: First, we resolved the GaN lattice in [1 100$]$ projection, which requires $0.15 \mathrm{~nm}$ of resolution. Second, the [1 100$]$ projection eliminates the stacking fault contrast that usually obscures lattice images recorded in [11이 projection. Thus, image interpretation is drastically simplified by simply having a choice of recording lattice images along different zone axes and yet resolving a lattice.

From a focal series of lattice images it is then possible to reconstruct the electron exit wave shown in figure 5a. Again, the reconstructed phase image is directly interpretable: a bright spot in the exit wave picture marks the position of atomic columns, its intensity depends on chemical composition and the spacing of atoms along the column (equation 1). It is seen that all atom columns at the interface and in the sapphire are resolvable with a smallest projected aluminum oxygen spacing of $85 \mathrm{pm}$ in the sapphire. Consequently, it is now possible to detect single columns of oxygen atoms at a sub Angstrom spacing because of the excellent signal to noise ratio of the phase contrast image and the improved resolution.

An additional advantage of phase contrast imaging comes from the precision as to which lattice parameters can be measured ${ }^{12)}$. Figure 5 b summarizes lattice parameter measurements in plane of the interface and normal (out of plane) to it. It is seen that the in plane lattice parameter does not vary more than $1 \mathrm{pm}$ except for a surprisingly $0.8-1 \mathrm{~nm}$ narrow interfacial region. Even the out of plane lattice parameter is reduced by only $10 \mathrm{pm}$ which corresponds to a displacement of only $4 \%$. One would estimate an out of plane lattice parameter variation of roughly $30 \%$ for fully strained pseudomorphic growth with a lattice mismatch of $14 \%$. Closer inspection of the narrow interfacial region reveals the presence of a misfit dislocation network. One of the misfit dislocations is depicted in figure 6. It is seen that its core structure is entirely resolved. The dislocation is of edge character and a row of oxygen or nitrogen atoms terminates the inserted extra half plane. It is not possible to discriminate between nitrogen and oxygen atoms because of their similar $\mathrm{Z}$ of 7 and 8, respectively. The misfit dislocation spacing along the interface is regular. From figure 5a we find that they are standing off the interface by $0.5-1 \mathrm{~nm}$ and that six

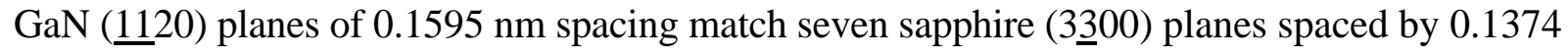
$\mathrm{nm}$. Here we used literature values for lattice parameters. Resultantly, one calculates that 0.957 $\mathrm{nm}$ in $\mathrm{GaN}$ match $0.962 \mathrm{~nm}$ in sapphire along the interface which leaves a residual strain of less 
than $1 \%$. Such values agree reasonably well with the measured $5 \%$ of the out of plane lattice parameter change considering that GaN growth can be largely non stoichiometric ${ }^{22)}$.

In summary, we applied phase contrast microscopy to investigate the interface between sapphire and $\mathrm{GaN}$ with sub Angstrom resolution. The investigation benefits from the gained ability to choose from different zone axes that comes with the improvement of resolution and the large sensitivity of phase contrast microscopy that enables scientists to detect light elements even if they are closely spaced to heavier next neighbors. Dislocation core structures are now resolvable. In this particular case we find that the complicated process to initiate growth of $\mathrm{GaN}$ on sapphire $^{22)}$ is linked to the fabrication of a misfit dislocation network that accommodates most of the initial lattice mismatch of $14 \%$. The dislocated area is confined to a $0.8-1 \mathrm{~nm}$ narrow interfacial region. Residual displacements are about 5\% and they vanish over several nanometers as the distance from the interface increases in the thin TEM foils.

\section{Conclusions}

Investigations of $\mathrm{GaN}$ on sapphire and of the silicon gate oxides are performed to analyze two technologically important materials with state-of-the-art Transmission Electron Microscopy. Phase contrast microscopy, Z-contrast microscopy, and electron spectroscopy are employed. In the case of silicon gate oxides a moderate resolution of $136 \mathrm{pm}$ suffices to resolve the structure which is achievable with all techniques these days. The presence of the amorphous gate dielectric, however, favors applications of Z-contrast microscopy because of frequency limitations that come with the application of phase contrast microscopy and produces a speckle contrast in the amorphous layer. It complicates a quantitative image analyses. The application of electron spectroscopy is desirable in any case. Here we demonstrate that industry seeks to increase switching times in Si based devices by increasing the dielectric constant of the gate oxide with the addition of nitrogen. Its diffusion can be controlled such that the $\mathrm{N}$ concentration peaks at the poly-Si side but is negligibly small on the $\mathrm{SiO}_{2} / \mathrm{Si}$ side of the gate oxide. In addition a nitridation of the gate oxide increases its width which reduces the risk of undesirable tunnel currents as the dimensions of the devices shrink. In case of GaN on Sapphire, only phase contrast microscopy with sub Angstrom resolution allows to resolve all atomic columns at this 
is now possible to systematically study the initiation of thin film growth on ceramic materials and semiconductors with short bond length on a truly atomic level. Overall the investigation demonstrates that newly emerging TEM techniques can be chosen such that they provide a best solution for a particular problem in materials sciences.

\section{Acknowledgement}

The project is sponsored by the Director, Office of Science, Office of Basic Energy Sciences, of the U.S. Department of Energy under Contract No. DE-AC03-76SF00098.

\section{References}

1. C. Kisielowski, E.C. Nelson, C. Song, R. Kilaas, A. Thust, Microscopy and Microanalysis 6 (2000) 16

2. M.A. O'Keefe, Microscopy and Microanalysis 6 (2000) 1192

3. C. Kisielowski, C.J.D. Hetherington, Y.C. Wang, R. Kilaas, M.A. O'Keefe, A. Thust, Ultramicroscopy (2001) in press

4. M.A. O'Keefe, C.J.D. Hetherington, Y.C. Wang, E.C. Nelson, J.H. Turner, C. Kisielowski, J.-O. Malm, R. Mueller, J. Ringnalda, M. Pam, A. Thust, Ultramicroscopy (2001) in press

5. W.M.J. Coene, A. Thust, M. Op de Beeck, D. Van Dyck, Ultramicroscopy 64 (1996) 109

6. A. Thust, W.M.J. Coene, M. Op de Beeck, D. Van Dyck, Ultramicroscopy 64 (1996) 211

7. P.D. Nellist, S.J. Pennycook, Phys. Rev. Letters 81 (1998) 4156

8. G. Duscher, M.Kim, N.D. Browning, S.T. Pantelides, S.J. Pennycook, Microscopy and Microanalysis, 6 (2000) 114

9. O.L. Krivanek, N. Dellby, A.R. Lupini, Ultramicroscopy 78 (1999) 1

10. P.E. Betson, , Ultramicroscopy 78 (1999) 33

11. P.C. Tiemeijer, , Ultramicroscopy 78 (1999)( 53

12. C. Kisielowski, O. Schmidt, J. Yang, Mat. Res. Soc. Symp., 482 (1998) 369

13. C.L. Jia, A. Thust, Phys. Rev. Lett. 82 (1999) 5052

14. S. Johnson, in "Strategies in Light 2001“, Strategies Unlimited, San Francisco 2001

15. D.A. Muller, T. Sorsch, S. Moccio, F.H. Baumann, K. Evans-Lutterodt, G. Timp, Nature 
16. D.B. Wiliams, C.B. Carter in „Transmission Electron Microscopy“, Plenum Press, New York 1996

17. D. van Dyck, J.H. Chen, Solid State Communications 109 (1999) 501

18. C.Kisielowski, J.M.Plitzko, S.Lartigue, T.Radetic, U.Dahmen, Microscopy and Microanalysis 7 (2001) 246

19. S.J. Pennycook, B. Rafferty, P.D. Nellist, Microscopy and Microanalysis 6 (2000) 106

20. E.M. James, N.D.Browning, Ultramicroscopy 78 (1999) 125

21. E.D. Boyes, J. Rignalda, M.A.J. van der Stam, T.F. Fliervoet, E. Van Cappellen, Microscopy and Microanalysis 7 (2001) 232

22. C. Kisielowski in „Semiconductors and Semimetals“ 57, J.I. Pankove and T.D. Moustakas eds., Academic Press, New York 1999, 257

\section{Table1:}

Quantitative evaluation of gate oxide thickness and interfacial roughness from Z-contrast and phase contrast images. Sample A: control sample. Sample B: nitrogen containing gate oxide. Roughness measurements were made from Z-contrast images.

$\begin{array}{ccccc}\text { Sample } & \text { Thickness }[\mathrm{nm}] & \text { Thickness }[\mathrm{nm}] & \text { Roughness }[\mathrm{nm}] & \text { Roughness }[\mathrm{nm}] \\ \text { Z-contrast } & \text { Phase contrast } & \mathrm{Si}^{\prime} \mathrm{SiO}_{2} & \text { Poly-Si/SiO }_{2} \\ \text { B } & 1.92 & 1.76 & 0.06 & 0.10 \\ \text { B } & 2.65 & 2.14 & 0.08 & 0.25 \\ \text { B } & 2.50 & 2.06 & 0.06 & 0.14 \\ & 2.61 & 2.00 & 0.07 & --\end{array}$

Figure captions 


\section{Figure 1}

Band gap energy versus inter atomic distances in selected semiconductors and ceramics. The slope of the trend line relates $1 \mathrm{pm}$ of distance to $50 \mathrm{meV}$ of energy change.

\section{Figure 2}

Gate oxides in silicon. Images were recorded by different techniques. a) A traditional lattice image recorded with NCEM's One Angstrom Microscope (OAM) that is a specially equipped Philips CM300. b) Reconstructed electron exit wave from a focus series of twenty lattice images of the OAM. The phase of the electron exit wave is shown. c) Scanning Transmission Electron Microscopy (STEM) was utilized to produce a Z-contrast image. A Tecnai F20 super twin microscope was used.

Figure 3

More than 1000 Electron Energy Loss Spectra were recorded with an electron beam focused to $0.19 \mathrm{~nm}$ small spot that was repetitively scanned across the gate oxide (Tecnai F20). An automated data analysis produces the depicted profiles of nitrogen, silicon and oxygen in minutes. A $2.07 \mathrm{~nm}$ wide dielectric was measured from image patterns in phase contrast images. An analysis of the local intensity of Z-contrast images reveals a 2.nm wide gate oxide.

\section{Figure 4}

Lattice images of GaN along two different zone axes a and b. An information limit of $80 \mathrm{pm}$ of the OAM allows for a choice of suitable zone axes. Here, they are chosen such that the contrast of stacking faults is eliminated in b. Insets show the unit cells of hexagonal GaN projected along the two different zones.

Figure 5 
Top a) Phase of the electron exit wave reconstructed from 20 lattice images. b) Measurement of lattice parameters across the field of view. Values in plane of the interface and normal to it (out of plane) are averaged in columns across the picture.

\section{Figure 6}

Core structure of an oxygen terminated misfit dislocation at the interface between $\mathrm{GaN}$ and sapphire. The model (right) was obtained from pinpointing atomic columns in the phase image. 


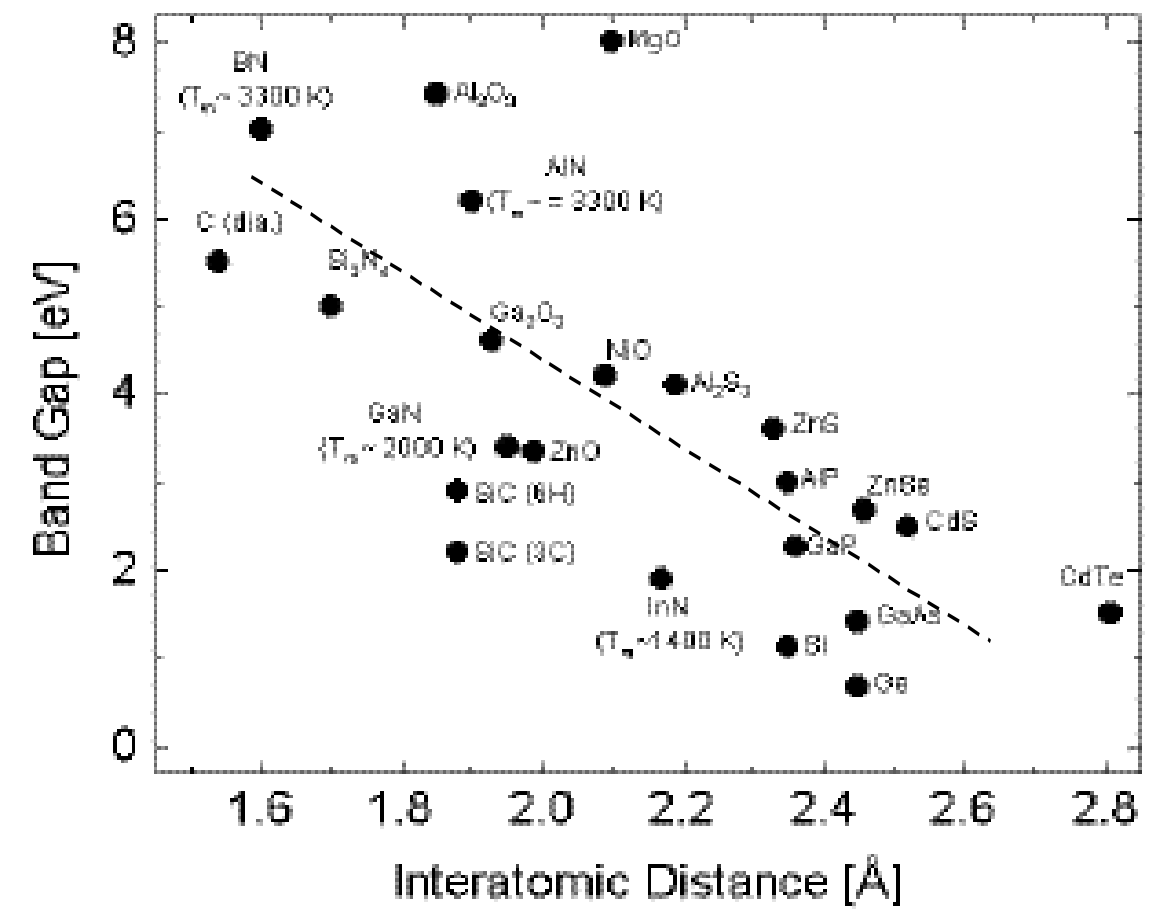

Figure 1 , C. Kisielowski et al. 


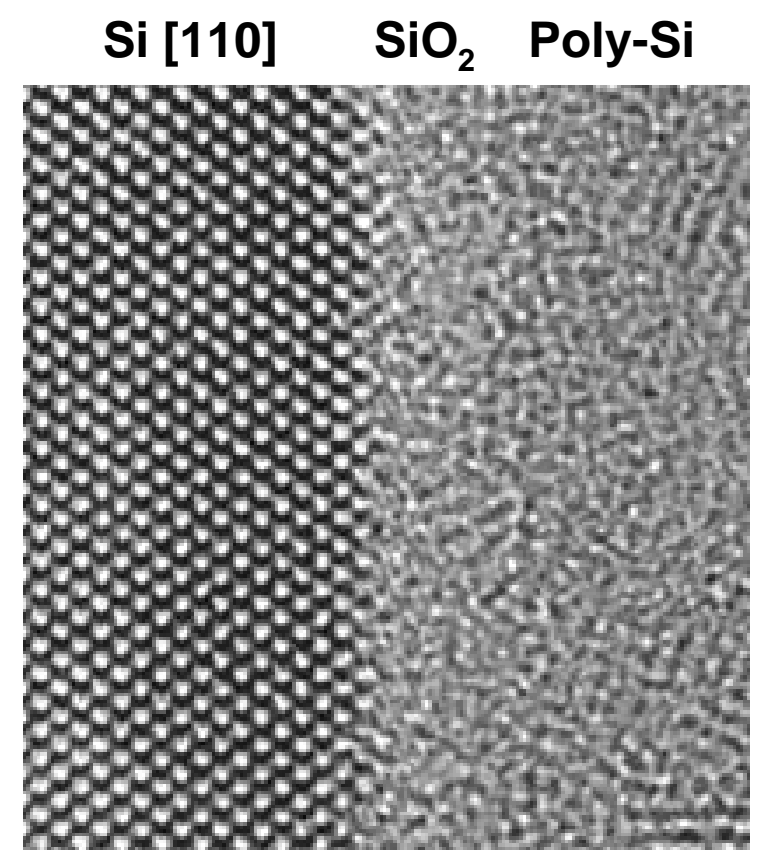

Lattice image (OAM)

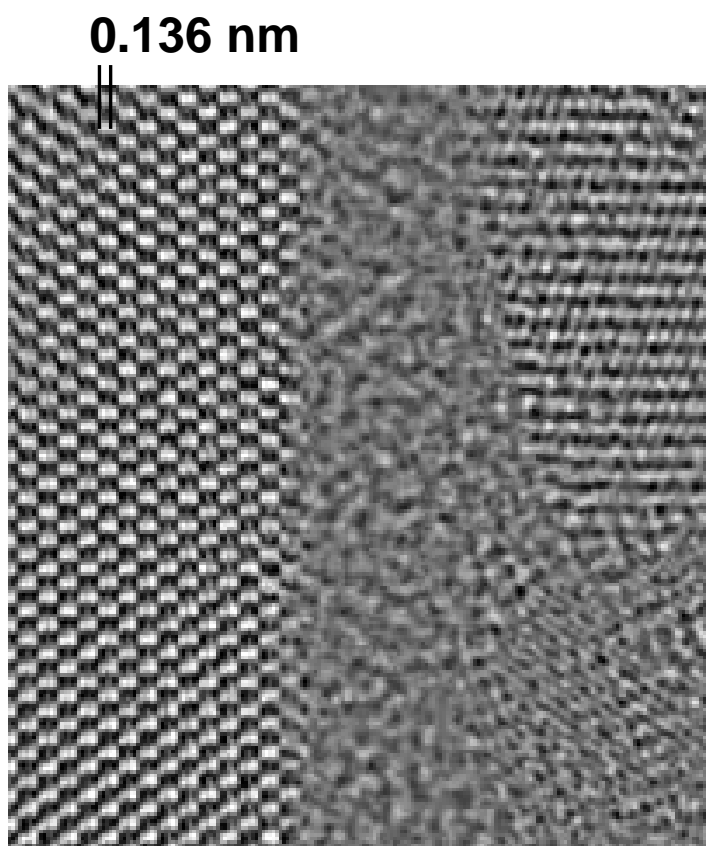

Reconstructed phase image (OAM)

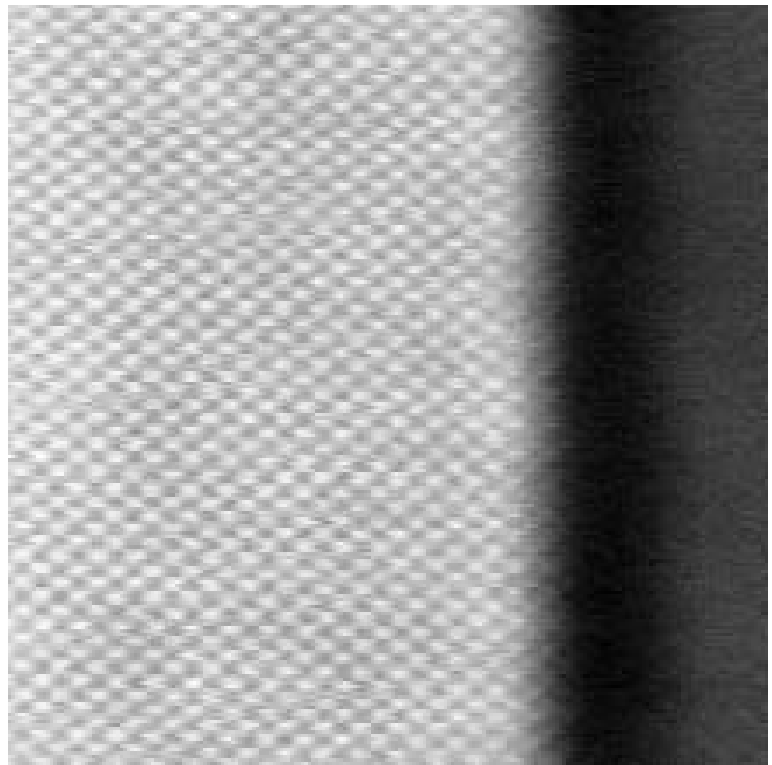

Z contrast image (Tecnai F20/ST) a)

Figure 2 , C. Kisielowski et al. b)

c) 


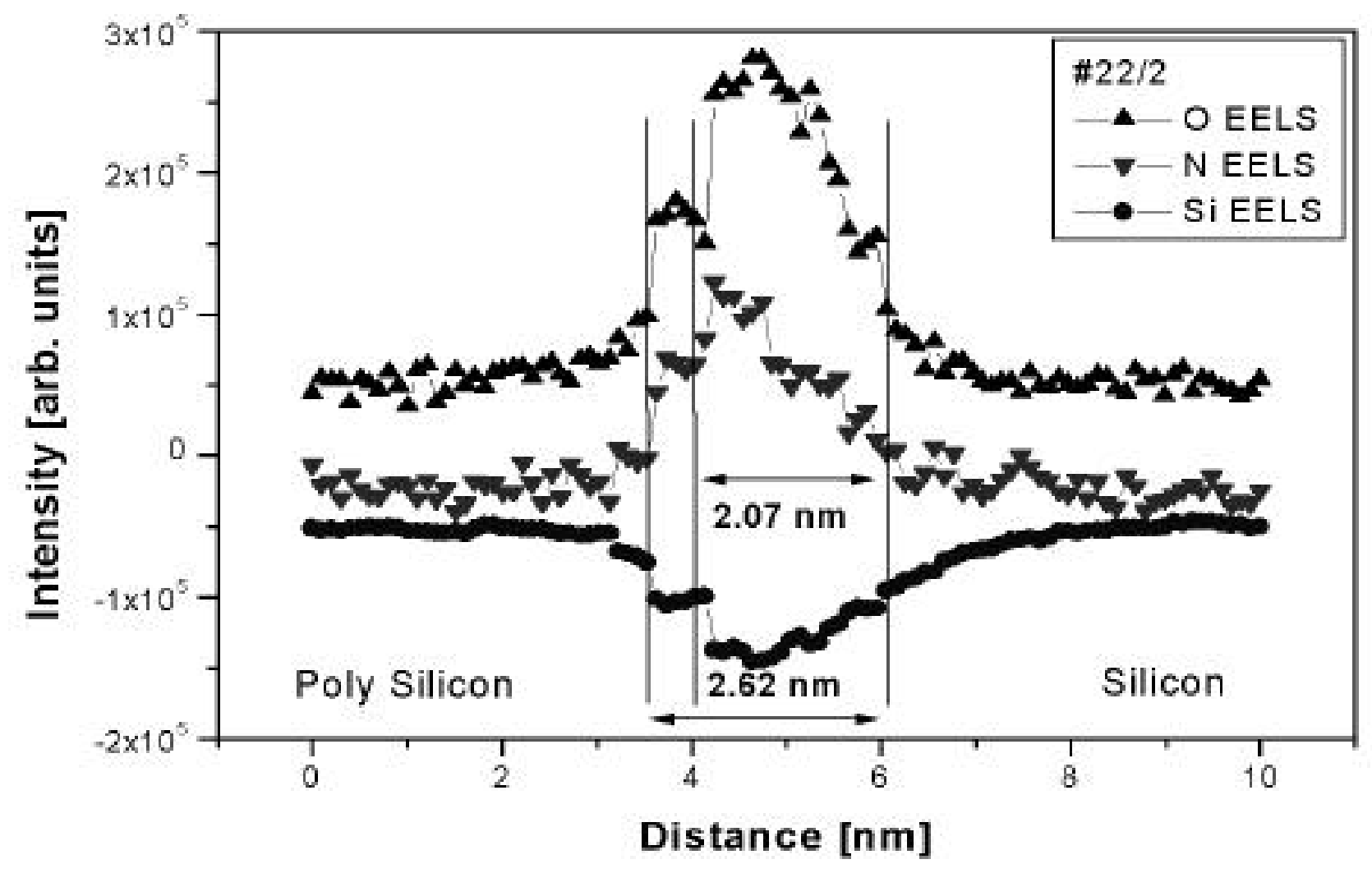

Figure 3, C. Kisielowski et al. 


\section{Zone axes:}

[1120]


Figure 4 , C. Kisielowski et al.

a)

b) 
a)

b)

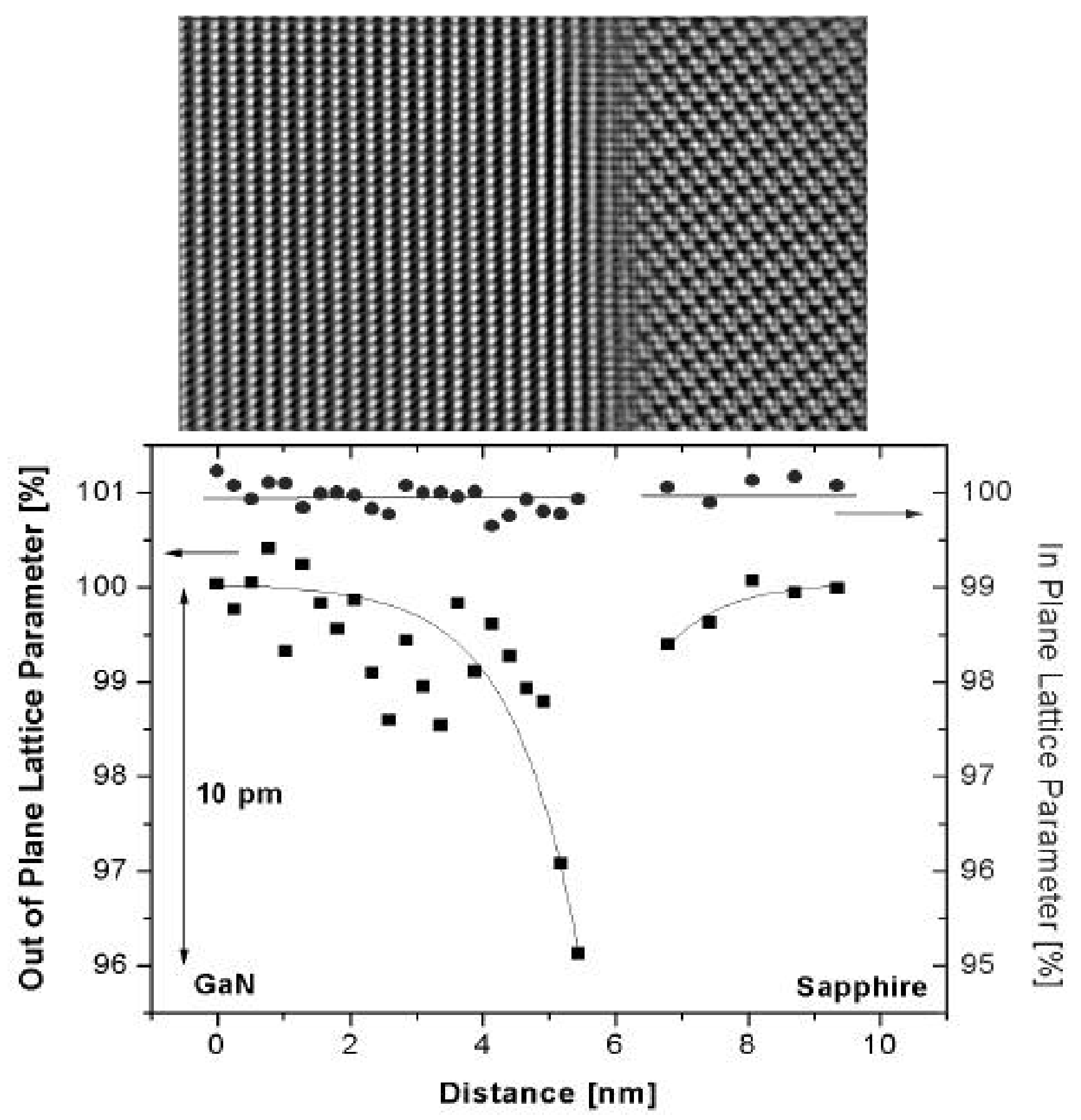

Figure 5, C. Kisielowski et al. 


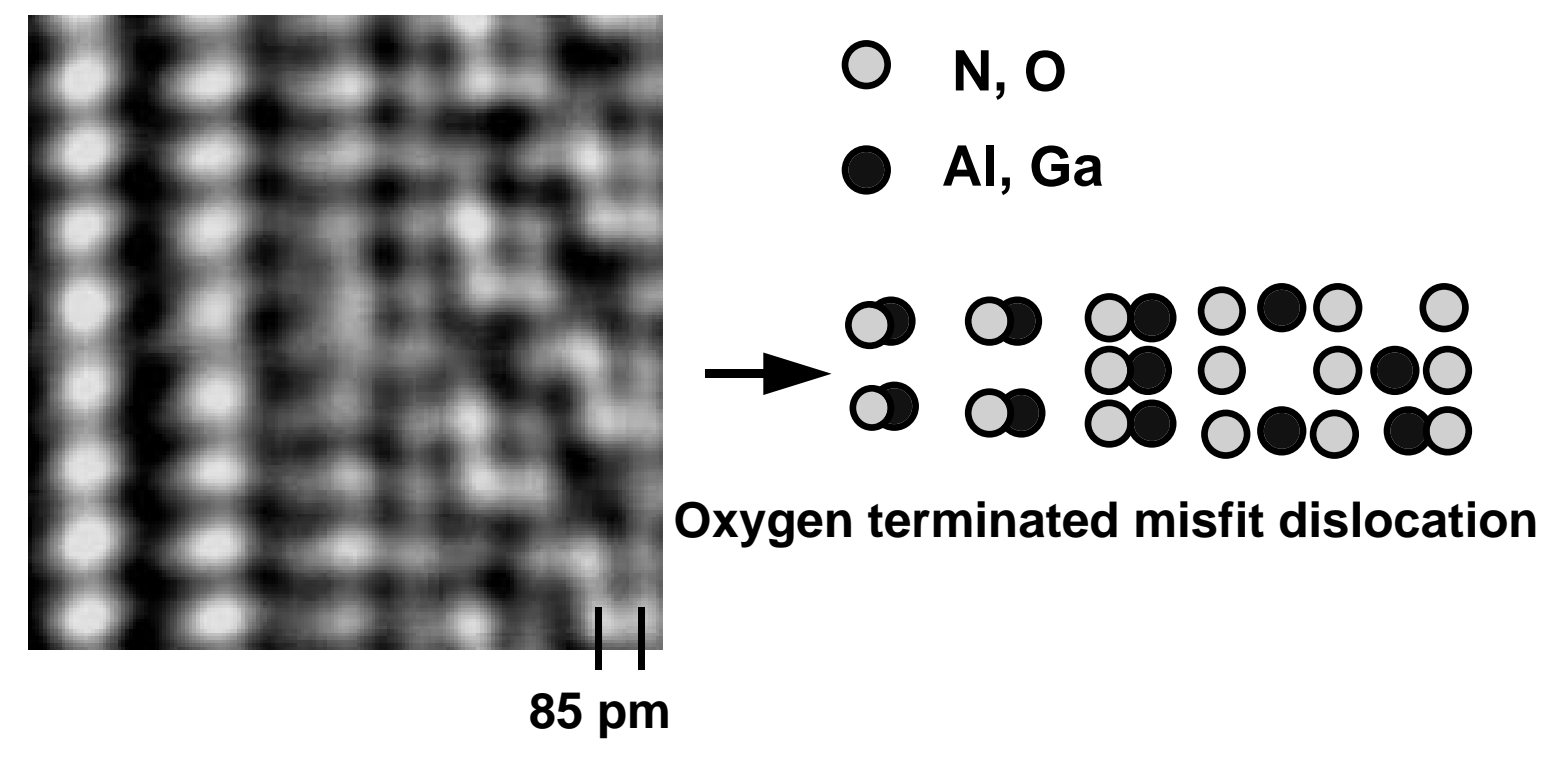

Figure 6, C. Kisielowski et al. 